\title{
Critical Values of Higher Derivatives of Twisted Elliptic L-Functions
}

\author{
Jack Fearnley and Hershy Kisilevsky
}

\section{CONTENTS}

1. Introduction

2. The Elliptic Regulator

3. The Generalized Dedekind-Frobenius Theorem

4. The Critical $L$-Value

5. Numerical Data

Acknowledgments

References
2000 AMS Subject Classification: 11G05, 11G40, 11Y40

Keywords: elliptic curves, $L$-functions
Let $L(E / \mathbb{Q}, s)$ be the $L$-function of an elliptic curve $E$ defined over the rational field $\mathbb{Q}$. Assuming the Birch-Swinnerton-Dyer conjectures, we examine special values of the $r$ th derivatives, $L^{(r)}(E, 1, \chi)$, of twists by Dirichlet characters of $L(E / \mathbb{Q}, s)$ when $L(E, 1, \chi)=\cdots=L^{(r-1)}(E, 1, \chi)=0$.

\section{INTRODUCTION}

Let $E / \mathbb{Q}$ be an elliptic curve defined over the field $\mathbb{Q}$. Denote by

$$
L(E / \mathbb{Q}, s)=\sum_{n \geq 1} a_{n} n^{-s}
$$

its $L$-function. If $K / \mathbb{Q}$ is a finite extension, then the Birch-Swinnerton-Dyer conjecture asserts that the first nonvanishing derivative satisfies

$$
\frac{L^{\left(r_{K}\right)}(E / K, 1)}{r_{K} !}=\frac{\Omega_{K}|\amalg(K)| R_{K}}{\sqrt{|d(K)|\left|E(K)_{\text {Tors }}\right|^{2}}} \prod_{\mathfrak{p}} c_{\mathfrak{p}},
$$

where $r_{K}=\operatorname{rank}_{\mathbb{Z}} E(K)$ and $\Omega_{K}$ is a product of periods of $E, d(K)$ is the discriminant of $K$, and $R_{K}$ denotes the elliptic regulator, i.e., $R_{K}$ is the absolute value of the determinant of the height-pairing matrix of $E(K)$. Also $\amalg(K)$ is the Tate-Shafarevich group of $E / K$, and the $c_{\mathfrak{p}}$ are the Tamagawa numbers of $E / K$. (See [Dokchitser and Dokchitser 10] for the computation of the Tamagawa numbers in extension fields.)

If $K / \mathbb{Q}$ is an abelian extension with $\operatorname{Gal}(K / \mathbb{Q})=G$, then there is a factorization

$$
L(E / K, s)=\prod_{\chi \in \widehat{G}} L(E, s, \chi)
$$

where $\widehat{G}$ is the group of primitive Dirichlet characters associated with the extension $K / \mathbb{Q}$. Then assuming the Birch-Swinnerton-Dyer conjecture, $r_{K}$ and $r_{\mathbb{Q}}$ are the orders of vanishing at $s=1$ of $L(E / K, s)$ and $L(E / \mathbb{Q}, s)$ respectively. Let $r_{\chi}$ denote the order of vanishing at $s=1$ 
of $L(E, s, \chi)$. Then

$$
\frac{L^{\left(r_{K}\right)}(E / K, 1)}{r_{K} !}=\frac{L^{\left(r_{\mathbb{Q}}\right)}(E / \mathbb{Q}, 1)}{r_{\mathbb{Q}} !} \cdot \prod_{\substack{\chi_{0} \neq \chi \in \widehat{G} \\ r_{\chi} !}} \frac{L^{\left(r_{\chi}\right)}(E, 1, \chi)}{r} .
$$

Therefore, assuming the Birch-Swinnerton-Dyer conjecture, it follows that

$$
\begin{aligned}
& \frac{\Omega_{K}|\amalg(K)| R_{K}}{\sqrt{|d(K)|\left|E(K)_{\text {Tors }}\right|^{2}}} \prod_{\mathfrak{p}} c_{\mathfrak{p}} \\
& =\frac{\Omega^{+}|\amalg(\mathbb{Q})| R_{\mathbb{Q}}}{\left|E(\mathbb{Q})_{\text {Tors }}\right|^{2}} \prod_{p} c_{p} \prod_{\chi_{0} \neq \chi \in \widehat{G}} \frac{L^{\left(r_{\chi}\right)}(E, 1, \chi)}{r_{\chi} !},
\end{aligned}
$$

where $\Omega^{+}$is the real period of $E$. If $\Omega^{-}$denotes the (positive) complex part of the imaginary period of $E$, then $\Omega_{K}=\left(\Omega^{+}\right)^{[K: \mathbb{Q}]}$ if $K$ is a totally real field and $\Omega_{K}=\left(\Omega^{+} \Omega^{-}\right)^{[K: \mathbb{Q}] / 2}$ if $K$ is a totally imaginary field.

The Deligne-Gross conjecture [Deligne 79, p. 323] implies that $r_{\chi}=r_{\chi^{\prime}}$ if $\chi^{\prime}$ and $\chi$ are Galois conjugate characters, i.e., if $\chi^{\prime}(\sigma)=\chi^{\gamma}(\sigma)=\gamma(\chi(\sigma))$ for some $\gamma \in$ $\operatorname{Gal}(\mathbb{Q}(\chi) / \mathbb{Q})$ and for all $\sigma \in G$.

In [Fearnley and Kisilevsky 10] we examine the case that $K / \mathbb{Q}$ is a cyclic extension of odd prime degree, $[K: \mathbb{Q}]=\ell$, and that the $\chi$-rank is one, i.e., that $r_{\chi}=1$ for all $\chi \neq \chi_{0}$, where $\chi_{0}$ denotes the principal or trivial character. In the present article, we consider the case that $K / \mathbb{Q}$ is a cyclic extension of odd prime degree, $[K: \mathbb{Q}]=\ell$, and that the $\chi$-rank $r_{\chi}=r$ is greater than one. That is, we suppose that

$$
L(E, 1, \chi)=\cdots=L^{\left(r_{\chi}-1\right)}(E, 1, \chi)=0
$$

and

$$
L^{\left(r_{\chi}\right)}(E, 1, \chi) \neq 0 .
$$

In Section 4 below, for a primitive Dirichlet character $\chi$ of conductor $\mathfrak{f}_{\chi}$, we propose an explicit formula for the value $L^{\left(r_{\chi}\right)}(E, 1, \chi)$, namely

$\frac{L^{\left(r_{\chi}\right)}(E, 1, \chi)}{r_{\chi} !}=\frac{\tau(\chi)}{\mathfrak{f}_{\chi}} \Omega^{+} \delta_{\chi}\left(P_{1}, \ldots, P_{r}\right) \alpha_{\chi}^{+}\left(P_{1}, \ldots, P_{r}\right) z_{\chi}$,

where $\left\{P_{1}, \ldots, P_{r}\right\} \in E_{\operatorname{Tr}}(K)$ are independent points of infinite order in $E(K)$ with trace 0 to $\mathbb{Q}, \delta_{\chi}\left(P_{1}, \ldots, P_{r}\right)$ is a determinant that will be described below, and where $\alpha_{\chi}^{+}\left(P_{1}, \ldots, P_{r}\right)$ and $z_{\chi}$ are algebraic numbers in $\mathbb{Q}(\chi)$.

In Section 5, we present some numerical computations that we offer as evidence for formula (1-2).

These results can be considered as evidence in support of the equivariant Tamagawa number conjectures as formulated in [Burns and Flach 01] and [Burns 10, Theorem 5.1.1(i)]. More supporting evidence can be found in [Bley 10].

\section{THE ELLIPTIC REGULATOR}

Let $K / \mathbb{Q}$ be a finite extension. As in [Fearnley and Kisilevsky 10], suppose that $P_{1}, P_{2}, \ldots, P_{t}$ generate a subgroup $E_{\{P\}} \subseteq E(K)$ of rank $t$. The regulator matrix and the regulator (determinant) are defined as

$$
\begin{aligned}
R M\left(E_{\{P\}}\right) & :=\left(\left\langle P_{i}, P_{j}\right\rangle\right)_{1 \leq i, j \leq t}, \\
R\left(E_{\{P\}}\right) & :=\left|\operatorname{det}\left(\left\langle P_{i}, P_{j}\right\rangle\right)\right|,
\end{aligned}
$$

where $\langle\cdot, \cdot\rangle$ denotes the canonical Néron-Tate height pairing on $E$.

If $E_{\{P\}}$ together with $E(K)_{\text {Tors }}$ generates all of $E(K)$, then $R\left(E_{\{P\}}\right)=R_{K}$, the regulator of $E$ over $K$.

If $Q_{1}, Q_{2}, \ldots, Q_{t}$ generate a subgroup $E_{\{Q\}}$ of finite index $m$ in $E_{\{P\}}$, then

$$
R\left(E_{\{Q\}}\right)=m^{2} R\left(E_{\{P\}}\right) .
$$

Therefore, passing to subgroups of finite index introduces only integral square factors in the regulators.

Let $E_{\mathrm{Tr}}(K)$ denote the set of points $P \in E(K)$ with trace zero to $E(\mathbb{Q})$, i.e.,

$$
E_{\operatorname{Tr}}(K)=\left\{P \in E(K) \mid \operatorname{Tr}_{K / \mathbb{Q}}(P)=\sum_{\sigma \in G} P^{\sigma}=0\right\} .
$$

Then it is shown in [Fearnley and Kisilevsky 10, Section 4] that $E_{\operatorname{Tr}}(K)$ is a subgroup of $E(K)$ that is orthogonal to $E(\mathbb{Q})$ with respect to the canonical Néron-Tate height pairing and such that the subgroup $E^{\prime}(K)$ of $E(K)$ generated by $E(\mathbb{Q})$ together with $E_{\mathrm{Tr}}(K)$ has finite index in $E(K)$. Consequently, the regulator matrix $R M\left(E^{\prime}(K)\right)$ has the form

$$
R M\left(E^{\prime}(K)\right)=\left(\begin{array}{cc}
R M(E(\mathbb{Q})) & 0 \\
0 & R M\left(E_{\operatorname{Tr}}(K)\right)
\end{array}\right) .
$$

Assume now that $K / \mathbb{Q}$ is a cyclic extension of odd prime degree $[K: \mathbb{Q}]=\ell \geq 3$, with Galois group $\operatorname{Gal}(K / \mathbb{Q})=G=\left\langle\sigma_{0}\right\rangle$. Then $E_{\operatorname{Tr}}(K)$ is a module for $\mathbb{Z}[G] /(\operatorname{Tr}) \simeq \mathbb{Z}\left[\zeta_{\ell}\right]$, the ring of integers in the cyclotomic field $\mathbb{Q}\left(\zeta_{\ell}\right)$ of $\ell$ th roots of unity. It is then shown in [Fearnley and Kisilevsky 10, Section 5] that there is a $G$-invariant subgroup $E^{*}(K) \subseteq E_{\operatorname{Tr}}(K)$ of finite index (bounded only in terms of $\ell$ ) such that

$$
E^{*}(K) \simeq(\mathbb{Z}[G] /(\operatorname{Tr}))^{r}
$$

for some integer $r \geq 0$. Therefore there exist points $P_{1}, \ldots, P_{r} \subset E^{*}(K)$ such that

$$
\left\{P_{1}, P_{1}^{\sigma_{0}}, \ldots, P_{1}^{\sigma_{0}{ }^{\ell-2}}, P_{2}, P_{2}^{\sigma_{0}}, \ldots, P_{r}, \ldots, P_{r}^{\sigma_{0}^{\ell-2}}\right\}
$$


is a (free) $\mathbb{Z}$-basis for $E^{*}(K)$. It follows that the group $E(\mathbb{Q}) \cdot E^{*}(K)$ has finite index (say $m$ ) in $E(K)$, and therefore the regulator $R_{K}$ factors as

$$
R_{K}=m^{2} \cdot R_{\mathbb{Q}} \cdot R\left(E^{*}(K)\right) .
$$

Since the canonical Néron-Tate height pairing satisfies $\left\langle P^{\sigma}, Q^{\sigma}\right\rangle=\langle P, Q\rangle$ for $P, Q \in E(K)$ and $\sigma \in G$, the matrix $R M\left(E^{*}\right)$ can be written as

$$
R M\left(E^{*}\right)=\left(\left\langle P_{i}^{\sigma}, P_{j}^{\tau}\right\rangle\right)=\left(\left\langle P_{i}, P_{j}^{\tau \sigma^{-1}}\right\rangle\right),
$$

where $1 \neq \sigma, \tau \in G$ and $1 \leq i, j \leq r$. In order to evaluate the regulator $R\left(E^{*}(K)\right)$, we define $r^{2}$ functions $f_{i, j}, 1 \leq$ $i, j \leq r$, as follows:

$$
\begin{aligned}
& f_{i, j}: G \longrightarrow \mathbb{C}, \\
& f_{i, j}(\sigma) \mapsto\left\langle P_{i}, P_{j}^{\sigma}\right\rangle \text { for } \sigma \in G .
\end{aligned}
$$

For $\chi \in \widehat{G}$, define

$$
\lambda_{\chi}\left(f_{i, j}\right)=\sum_{\sigma \in G} \chi(\sigma)\left\langle P_{i}, P_{j}^{\sigma}\right\rangle=\sum_{\sigma \in G} \chi(\sigma) f_{i, j}(\sigma) .
$$

If $\Lambda_{\chi}$ denotes the $r \times r$ matrix

$$
\Lambda_{\chi}=\left(\lambda_{\chi}\left(f_{i, j}\right)\right)
$$

let

$$
\delta_{\chi}=\operatorname{det} \Lambda_{\chi}
$$

Then it is proved in Theorem 3.1 below that

$$
R\left(E^{*}(K)\right)=\frac{1}{\ell^{r}} \prod_{\chi_{0} \neq \chi \in \widehat{G}} \delta_{\chi} .
$$

The factorizations (2-1) and (2-2) allow us to decompose the regulator $R_{K}$ (up to some rational factors bounded in terms of $\ell$ ) as a product

$$
R_{K}=q \cdot R_{\mathbb{Q}} \cdot \prod_{\chi_{0} \neq \chi \in \widehat{G}} \delta_{\chi}
$$

for some rational number $q$. The factors $\delta_{\chi}$ will appear in the critical $L$-values

$$
\frac{L^{\left(r_{\chi}\right)}(E, 1, \chi)}{r_{\chi} !}
$$

appearing in $(1-1)$.

\section{THE GENERALIZED DEDEKIND-FROBENIUS THEOREM}

In this section we derive a slight generalization of the Dedekind-Frobenius theorem for evaluating the determinant of the group matrix of an abelian group $G$. We follow the proof as found in [Washington 82].

\subsection{The Dedekind-Frobenius Theorem}

We suppose that $G$ is an abelian group of order $|G|=n$, whose elements will be denoted by $\sigma, \tau, \ldots$ Let $f: G \longrightarrow$ $\mathbb{C}$ be a complex-valued function on $G$. A group matrix is an $n \times n$ matrix $A=A(f)$ whose rows and columns are indexed by elements of $G$ (in some fixed order) and whose $(\sigma, \tau)$ entry is

$$
A_{\sigma, \tau}=f\left(\sigma^{-1} \tau\right)
$$

Then the Dedekind-Frobenius theorem states that

$$
\operatorname{det} A=\prod_{\chi \in \widehat{G}} \lambda_{\chi}(f)
$$

where $\widehat{G}$ is the character group of $G$, and for $\chi \in \widehat{G}$,

$$
\lambda_{\chi}(f)=\sum_{\sigma \in G} \chi(\sigma) f(\sigma)
$$

We note that $\left\{\lambda_{\chi}(f) \mid \chi \in \widehat{G}\right\}$ are the eigenvalues of the matrix $A$ with corresponding eigenvectors $\left\{\mathbf{w}_{\chi} \in \mathbb{C}^{n}\right.$ $\chi \in \widehat{G}\}$, where $\mathbf{w}_{\chi}$ is the vector such that the $\sigma$ coordinate satisfies $\left(\mathbf{w}_{\chi}\right)_{\sigma}=\chi(\sigma)$.

\subsection{The Generalized Dedekind-Frobenius Theorem}

In the present case, we consider, for an integer $r>0$, a collection $\mathcal{F}$ of $r^{2}$ complex-valued functions $f_{i, j}: G \longrightarrow$ $\mathbb{C}$ with $1 \leq i, j \leq r$. We then consider the $N \times N$ generalized group matrix $A=A(\mathcal{F})$, where $N=n \times r$ and the rows are indexed by pairs $(i, \sigma), 1 \leq i \leq r, \sigma \in G$, and columns $(j, \tau), 1 \leq j \leq r, \tau \in G$, and whose $((i, \sigma),(j, \tau))$ entry is

$$
A_{(i, \sigma),(j, \tau)}=f_{i, j}\left(\sigma^{-1} \tau\right)
$$

(If we fix some order on the the elements of $G$, then we may order the rows and columns lexicographically by stipulating that $(i, \sigma)<(j, \tau)$ whenever $i<j$, and if $i=$ $j$, then $\sigma<\tau$.)

We are also interested in the $(N-r) \times(N-r)$ matrices $C$ and $R$ with rows indexed by $(i, \sigma), 1 \leq i \leq r$, $1 \neq \sigma \in G$, and columns $(j, \tau), 1 \leq j \leq r, 1 \neq \tau \in G$, and whose entries are

$$
\begin{aligned}
& (C)_{(i, \sigma),(j, \tau)}=f_{i, j}\left(\sigma^{-1} \tau\right)-f_{i, j}(\tau), \\
& (R)_{(i, \sigma),(j, \tau)}=f_{i, j}\left(\sigma^{-1} \tau\right) .
\end{aligned}
$$

The matrix $A$ can be decomposed into $r^{2}$ blocks $A=$ $\left(A_{i, j}\right), 1 \leq i, j \leq r$, where $A_{i, j}$ is the $n \times n$ group matrix $A\left(f_{i, j}\right)$ whose $(\sigma, \tau)$ entry is

$$
\left(A_{i, j}\right)_{\sigma . \tau}=f_{i, j}\left(\sigma^{-1} \tau\right)
$$


Then $A_{i, j}=A\left(f_{i, j}\right)$ has eigenvalues $\left\{\lambda_{\chi}\left(f_{i, j}\right) \mid \chi \in \widehat{G}\right\}$ with corresponding common eigenvectors $\left\{\mathbf{w}_{\chi} \in \mathbb{C}^{n} \mid\right.$ $\chi \in \widehat{G}\}$, i.e., $A_{i, j} \mathbf{w}_{\chi}=\lambda_{\chi}\left(f_{i, j}\right) \mathbf{w}_{\chi}$ for all $1 \leq i, j \leq r$.

Define $\delta_{\chi}=\delta_{\chi}(\mathcal{F})$ as the determinant of the $r \times r$ ma$\operatorname{trix} \Lambda_{\chi}=\left(\lambda_{\chi}\left(f_{i, j}\right)\right)$ :

$$
\delta_{\chi}=\operatorname{det}\left(\Lambda_{\chi}\right) .
$$

Theorem 3.1. (Generalized Dedekind-Frobenius theorem.) Let $G$ be an abelian group and let $\mathcal{F}=\left\{f_{i, j} \mid 1 \leq\right.$ $i, j \leq r\}$ be a family of complex-valued functions on $G$. Then

1. the determinant of the $N \times N$ generalized group matrix $A=A(\mathcal{F})$ is

$$
\operatorname{det} A=\prod_{\chi \in \widehat{G}} \delta_{\chi}(\mathcal{F})
$$

2. the determinant of the $(N-r) \times(N-r)$ matrix $C$ is

$$
\operatorname{det} C=\prod_{\chi_{0} \neq \chi \in \widehat{G}} \delta_{\chi}(\mathcal{F})
$$

3. if for all pairs $(i, j)$ we have $\sum_{\sigma \in G} f_{i, j}(\sigma)=0$, the determinant of the $(N-r) \times(N-r)$ matrix $R$ is

$$
\operatorname{det} R=\frac{1}{|G|^{r}} \prod_{\chi_{0} \neq \chi \in \widehat{G}} \delta_{\chi}(\mathcal{F}) .
$$

Remark 3.2. The complex field $\mathbb{C}$ can be replaced by any field containing $n$ distinct $n$th roots of unity.

Proof. Let $\left\{\sigma_{1}=1, \sigma_{2}, \ldots, \sigma_{n}\right\}$ be a fixed ordering of the elements of $G$, and let $\left\{\chi_{1}=\chi_{0}, \chi_{2}, \ldots, \chi_{n}\right\}$ be a fixed ordering of the elements of $\widehat{G}$, where $\chi_{1}=\chi_{0}$ is the principal or trivial character of $G$. Let $V=\mathbb{C}^{N}$ and let $\mathcal{B}=\left\{\mathbf{e}_{i, \sigma} \mid 1 \leq i \leq r, \sigma \in G\right\}$ be the standard (lexicographically ordered) basis for $V$, where $\mathbf{e}_{i, \sigma}$ is the vector all of whose entries are 0 except for 1 in row $(i, \sigma)$.

Let $T: V \longrightarrow V$ denote the linear transformation on $V$ whose matrix with respect to the standard basis $\mathcal{B}$ is A. Then

$$
T\left(\mathbf{e}_{j, \tau}\right)=\sum_{(i, \sigma)} f_{i, j}\left(\sigma^{-1} \tau\right) \mathbf{e}_{i, \sigma} .
$$

The block decomposition of $A=\left(A_{i, j}\right)$ corresponds to the decomposition $V=\bigoplus_{i} W_{i}$, where $W_{i} \simeq \mathbb{C}[G]$ is the n-dimensional subspace of $V$ with basis $\mathcal{B}_{i}=\left\{\mathbf{e}_{i, \sigma} \mid \sigma \in\right.$ $G\}$.
But the space $W_{i}$ also has the ordered basis

$$
\mathcal{B}_{i}^{\prime}=\left\{\mathbf{v}_{i, \chi} \mid \chi \in \widehat{G}\right\},
$$

where

$$
\left(\mathbf{v}_{i, \chi}\right)_{j, \tau}= \begin{cases}0 & \text { if } j \neq i \\ \chi(\tau) & \text { if } j=i\end{cases}
$$

Then

$$
T\left(\mathbf{v}_{j, \chi}\right)=\sum_{i=1}^{r} \lambda_{\chi}\left(f_{i, j}\right) \mathbf{v}_{i, \chi}
$$

It follows that if we let $\mathcal{B}^{\prime}$ be the lexicographically ordered basis $\mathcal{B}^{\prime}=\cup_{i=1}^{r} \mathcal{B}_{i}^{\prime}$, then the matrix $A^{\prime}$ of $T$ with respect to $\mathcal{B}^{\prime}$ becomes a block matrix with $r \times r$ blocks each of which is diagonal. See Figure 1.

If we rearrange the elements of $\mathcal{B}^{\prime}$ as

$$
\mathcal{B}^{\prime \prime}=\left\{\mathbf{v}_{1, \chi_{1}}, \mathbf{v}_{2, \chi_{1}}, \ldots, \mathbf{v}_{r, \chi_{1}}, \ldots, \mathbf{v}_{1, \chi_{n}}, \ldots, \mathbf{v}_{r, \chi_{n}}\right\}
$$

and let $V^{\chi} \subseteq V$ be the $r$-dimensional subspace of $V$ with basis $\left\{\mathbf{v}_{i, \chi} \mid 1 \leq i \leq r\right\}$, then $V^{\chi}$ is a $T$-invariant subspace of $V$, and the matrix of $T$ with respect to $\mathcal{B}^{\prime \prime}$ becomes a block diagonal matrix with $r \times r$ blocks,

$$
A^{\prime \prime}=\left(\begin{array}{cccc}
\Lambda_{\chi_{1}} & & & \\
& \Lambda_{\chi_{2}} & & \\
& & \ddots & \\
& & & \Lambda_{\chi_{n}}
\end{array}\right),
$$

where $\Lambda_{\chi}=\left(\lambda_{\chi}\left(f_{i, j}\right)\right)$ is the matrix representing the restriction of $T$ to the subspace $V^{\chi}$ with respect to the basis $\left\{\mathbf{v}_{i, \chi} \mid 1 \leq i \leq r\right\}$.

It now follows that

$$
\operatorname{det} A=\prod_{\chi \in \widehat{G}} \operatorname{det} \Lambda_{\chi}=\prod_{\chi \in \widehat{G}} \delta_{\chi} .
$$

To prove statement 2 , we note that the space $W_{i}$ can be written as $W_{i}=W_{i}^{1} \oplus W_{i}^{0}$, where $W_{i}^{1}=\mathbb{C} \mathbf{v}_{i, \chi_{0}}, \mathbf{v}_{i, \chi_{0}}=$ $\sum_{\sigma \in G} \mathbf{e}_{i, \sigma}$, and

$$
W_{i}^{0}=\left\{\mathbf{w} \in W_{i} \mid \mathbf{w}=\sum_{\sigma \in G} x_{i, \sigma} \mathbf{e}_{i, \sigma} \text { and } \sum_{\sigma \in G} x_{i, \sigma}=0\right\} .
$$

Let $V^{0}=\bigoplus_{i=1}^{r} W_{i}^{0}$ and $V^{1}=\bigoplus_{i=1}^{r} W_{i}^{1}=V^{\chi_{0}}$. Since $\mathbf{v}_{i, \chi}=\sum_{\sigma \in G} \chi(\sigma) \mathbf{e}_{i, \sigma}$, we see that for $\chi \neq \chi_{0}$, we have $\mathbf{v}_{i, \chi} \in V^{0}$. Therefore if $\chi \neq \chi_{0}$, we have $V^{\chi} \subseteq V^{0}$, and counting dimensions, we find that

$$
V^{0}=\bigoplus_{\chi \neq \chi_{0}} V^{\chi}
$$




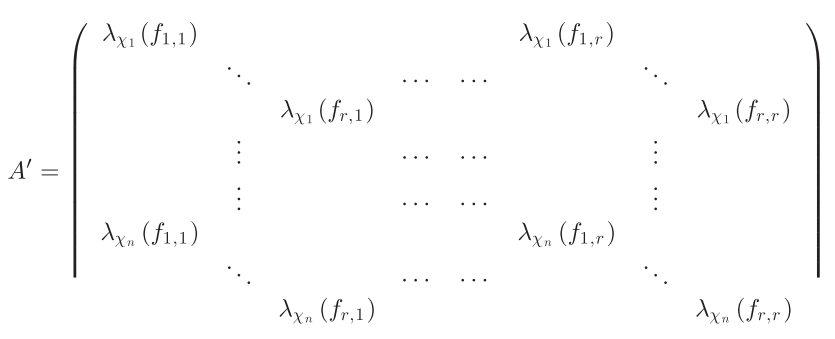

FIGURE 1. The matrix $A^{\prime}$ of $T$ with respect to $\mathcal{B}^{\prime}$ becomes a block matrix with $r \times r$ blocks each of which is diagonal.

It follows that $V^{0}$ is a $T$-invariant subspace of $V$, and that the determinant of $T$ restricted to $V^{0}$ is

$$
\operatorname{det}\left(T \mid V^{0}\right)=\prod_{\chi \neq \chi_{0}} \delta_{\chi}
$$

We now compute this determinant using another basis for $V^{0}$. Let

$$
\mathbf{f}_{i, \sigma}=\mathbf{e}_{i, \sigma}-\frac{1}{n} \mathbf{v}_{i, \chi_{0}}
$$

Then

$$
\mathbf{f}_{i, \sigma} \in W_{i}^{0}, \quad \sum_{\sigma \in G} \mathbf{f}_{i, \sigma}=0,
$$

and bases for $W_{i}$ and $W_{i}^{0}$ are respectively

$$
\left\{\mathbf{f}_{i, \sigma} \mid 1 \neq \sigma \in G\right\} \cup\left\{\mathbf{v}_{i, \chi_{0}}\right\}, \quad\left\{\mathbf{f}_{i, \sigma} \mid 1 \neq \sigma \in G\right\} .
$$

Computing $T \mid V^{0}$ with respect to the basis $\mathcal{C}=\left\{\mathbf{f}_{i, \sigma} \mid\right.$ $1 \leq i \leq r, 1 \neq \sigma \in G\}$, we obtain

$$
\begin{aligned}
T\left(\mathbf{f}_{j, \tau}\right) & =T\left(\mathbf{e}_{j, \tau}\right)-\frac{1}{n} T\left(\mathbf{v}_{j, \chi_{0}}\right) \\
& =\sum_{i, \sigma} f_{i, j}\left(\sigma^{-1} \tau\right) \mathbf{e}_{i, \sigma}-\frac{1}{n} \sum_{i} \lambda_{\chi_{0}}\left(f_{i, j}\right) \mathbf{v}_{i, \chi_{0}} \\
& =\sum_{i, \sigma}\left(f_{i, j}\left(\sigma^{-1} \tau\right)-\frac{1}{n} \lambda_{\chi_{0}}\left(f_{i, j}\right)\right) \mathbf{e}_{i, \sigma} .
\end{aligned}
$$

For fixed $(j, \tau)$, let $x_{i, \sigma}=\left(f_{i, j}\left(\sigma^{-1} \tau\right)-\frac{1}{n} \lambda_{\chi_{0}}\left(f_{i, j}\right)\right)$. Then since $T\left(\mathbf{f}_{j, \tau}\right) \in V^{0}$, we have $\sum_{\sigma \in G} x_{i, \sigma}=0$. Noting that $\mathbf{e}_{i, \sigma}=\mathbf{f}_{i, \sigma}+\frac{1}{n} \mathbf{v}_{i, \chi_{0}}$ and that $\sum_{\sigma} \mathbf{f}_{i, \sigma}=0$, we therefore have

$$
\begin{aligned}
T\left(\mathbf{f}_{j, \tau}\right) & =\sum_{i, \sigma} x_{i, \sigma}\left(\mathbf{f}_{i, \sigma}+\frac{1}{n} \mathbf{v}_{i, \chi_{0}}\right)=\sum_{i, \sigma} x_{i, \sigma} \mathbf{f}_{i, \sigma} \\
& =\sum_{i, 1 \neq \sigma} x_{i, \sigma} \mathbf{f}_{i, \sigma}+\sum_{i} x_{i, 1} \mathbf{f}_{i, 1} \\
& =\sum_{i, 1 \neq \sigma}\left(x_{i, \sigma}-x_{i, 1}\right) \mathbf{f}_{i, \sigma} \\
& =\sum_{i, 1 \neq \sigma}\left(f_{i, j}\left(\sigma^{-1} \tau\right)-f_{i, j}(\tau)\right) \mathbf{f}_{i, \sigma} .
\end{aligned}
$$

Let $C$ denote the matrix for $T \mid V^{0}$ with respect to the basis $\mathcal{C}$. Then $C$ is an $(N-r) \times(N-r)$ matrix indexed by rows $(i, \sigma), 1 \leq i \leq r, 1 \neq \sigma \in G$, and columns $(j, \tau)$, $1 \leq j \leq r, 1 \neq \tau \in G$. Then as in part one of the proof, $C$ has a block decomposition

$$
C=\left(C_{i, j}\right),
$$

where the $(\sigma, \tau)$ entry of $C_{i, j}$ is

$$
C_{(i, \sigma),(j, \tau)}=\left(f_{i, j}\left(\sigma^{-1} \tau\right)-f_{i, j}(\tau)\right) .
$$

Thus we have

$$
\operatorname{det} C=\prod_{\chi_{0} \neq \chi \in \widehat{G}} \delta_{\chi} .
$$

To prove the third statement, let $C^{*}=\left(C_{i, j}^{*}\right)$ be the augmented $N \times N$ block matrix with blocks

$$
C_{i, j}^{*}=\left(\begin{array}{cc}
\delta_{i, j} & f_{i, j}(\tau) \\
0 & C_{i, j}
\end{array}\right),
$$

where $\delta_{i, j}$ is the Kronecker delta function. By simultaneously permuting the rows and columns of $C^{*}$ so that the rows and columns indexed by $(i, 1)$ and $(1, j)$ appear in the upper left-hand corner, we obtain that $C^{*}$ is similar to the matrix

$$
B=\left(\begin{array}{ll}
I_{r} & * \\
0 & C
\end{array}\right),
$$

where $I_{r}$ is the $r \times r$ identity matrix. Therefore

$$
\operatorname{det} C^{*}=\operatorname{det} B=\prod_{\chi_{0} \neq \chi \in \widehat{G}} \delta_{\chi} .
$$

In the matrix $B$, we add row $(i, 1)$ to all the rows indexed by $(i, \sigma), \sigma \neq 1$, and then add all the resulting rows indexed by $(i, \sigma), \sigma \neq 1$, back to row $(i, 1)$. Using the fact that $\sum_{\sigma \in G} f_{i, j}(\sigma)=0$, we obtain the matrix

$$
B^{\prime}=\left(\begin{array}{cc}
n I & 0 \\
* & R
\end{array}\right),
$$

where $R$ is the $(N-r) \times(N-r)$ matrix with rows indexed by $(i, \sigma), 1 \leq i \leq r, 1 \neq \sigma \in G$, and columns $(j, \tau)$, 
$1 \leq j \leq r, 1 \neq \tau \in G$, and whose entries are

$$
(R)_{(i, \sigma),(j, \tau)}=f_{i, j}\left(\sigma^{-1} \tau\right) .
$$

Since $\operatorname{det}\left(B^{\prime}\right)=\operatorname{det}(B)=\operatorname{det}(C)$, it follows that

$$
\operatorname{det} R=\frac{1}{|G|^{r}} \prod_{\chi_{0} \neq \chi \in \widehat{G}} \delta_{\chi}(\mathcal{F}) .
$$

\section{THE CRITICAL L-VALUE}

In this section, let $E / \mathbb{Q}$ be an elliptic curve and let $K / \mathbb{Q}$ be a cyclic extension of odd prime degree $\ell$ and Galois group $\operatorname{Gal}(K / \mathbb{Q})=G$. For each character $\chi \in \widehat{G}$, we propose, assuming the Birch-SwinnertonDyer conjecture and the Deligne-Gross conjecture, a formula for the critical $L$-value $L^{\left(r_{\chi}\right)}(E, 1, \chi)$. The Deligne-Gross conjecture implies that $r_{\chi}=r$ is independent of $\chi$ for $\chi \neq \chi_{0}$, and by definition of $r_{\chi}$, we have

$$
\begin{aligned}
L(E, 1, \chi) & =\cdots=L^{\left(r_{\chi}-1\right)}(E, 1, \chi)=0, \\
L^{\left(r_{\chi}\right)}(E, 1, \chi) & \neq 0 .
\end{aligned}
$$

Also let $\tau(\chi)$ denote the Gauss sum

$$
\tau(\chi)=\sum_{a=0}^{\mathfrak{f}_{\chi}-1} \chi(a) \exp \left(2 \pi i a / \mathfrak{f}_{\chi}\right) .
$$

In [Fearnley and Kisilevsky 10, Proposition 3.1], it is shown that if we choose

$$
z_{\chi}= \begin{cases}1+\zeta & \text { if } \zeta=(-1)^{r_{\chi}} w_{E} \chi\left(N_{E}\right) \neq-1 \\ \chi\left(\sigma_{0}\right)-\bar{\chi}\left(\sigma_{0}\right) & \text { if } \zeta=(-1)^{r_{\chi}} w_{E} \chi\left(N_{E}\right)=-1\end{cases}
$$

where $w_{E}$ is the root number of $E / \mathbb{Q}$ and $N_{E}$ is the conductor of $E$, then we have

$$
\frac{\mathfrak{f}_{\chi}}{\tau(\chi) \Omega^{+} z_{\chi}} \frac{L^{\left(r_{\chi}\right)}(E, 1, \chi)}{r_{\chi} !} \in \mathbb{R}
$$

Summarizing, by [Fearnley and Kisilevsky 10, Section $5]$ and the discussion in Section 2, there are $r=r_{\chi}$ points $P_{1}, \ldots, P_{r}$ belonging to $E_{\mathrm{Tr}}(K)$ such that

$$
\left\{P_{i}^{\sigma} \mid 1 \leq i \leq r, 1 \neq \sigma \in G\right\}
$$

is a $\mathbb{Z}$-basis for a subgroup $E_{\{P\}}$ of finite index in $E_{\mathrm{Tr}}(K)$. For this choice, we define $\delta_{\chi}\left(P_{1}, \ldots, P_{r}\right)$ as in Section 2, and define $\alpha_{\chi}^{+}\left(P_{1}, \ldots, P_{r}\right)$ by

$$
\begin{aligned}
& \alpha_{\chi}^{+}\left(P_{1}, \ldots, P_{r}\right) \\
& \quad:=\frac{\mathfrak{f}_{\chi}}{\tau(\chi) \Omega^{+} z_{\chi} \delta_{\chi}\left(P_{1}, \ldots, P_{r}\right)} \frac{L^{\left(r_{\chi}\right)}(E, 1, \chi)}{r_{\chi} !} .
\end{aligned}
$$

If $\left\{Q_{i}^{\sigma} \mid 1 \leq i \leq r, 1 \neq \sigma \in G\right\}$ is another set of points that forms a $\mathbb{Z}$-basis for a subgroup $E_{\{Q\}}$ of finite index in $E_{\operatorname{Tr}}(K)$, then $\alpha_{\chi}^{+}\left(Q_{1}, \ldots, Q_{r}\right)$ differs from $\alpha_{\chi}^{+}\left(P_{1}, \ldots, P_{r}\right)$ by a factor that is the square of an algebraic number in $\mathbb{Q}(\chi)$. To see this, choose an integer, $b$ say, such that $b E_{\{Q\}} \subseteq E_{\{P\}}$. Therefore, for each $i, 1 \leq i \leq r$, we have

$$
b Q_{i}=\sum_{j=1}^{r} \xi_{i, j} P_{j}
$$

for some elements $\xi_{i, j} \in \mathbb{Z}[G]$. For $\xi=\sum_{\sigma \in G} a_{\sigma} \sigma \in \mathbb{Z}[G]$, define $\chi(\xi)=\sum_{\sigma \in G} a_{\sigma} \chi(\sigma)$.

Then using the relation

$$
\lambda_{\chi}(\xi(P), \eta(Q))=\chi(\xi) \bar{\chi}(\eta) \lambda_{\chi}(P, Q),
$$

[Fearnley and Kisilevsky 10, Corollary 6.2], we find that

$$
\delta_{\chi}\left(Q_{1}, \ldots, Q_{r}\right)=\frac{\left|\operatorname{det} \Xi_{\chi}\right|^{2}}{b^{2 r}} \delta_{\chi}\left(P_{1}, \ldots, P_{r}\right),
$$

where $\Xi_{\chi}$ is the $r \times r$ matrix

$$
\Xi_{\chi}=\left(\chi\left(\xi_{i, j}\right)\right) \text {. }
$$

Since

$$
\frac{L^{\left(r_{\chi}\right)}(E, 1, \chi)}{r_{\chi} !}=\frac{\tau(\chi)}{\mathfrak{f}_{\chi}} \Omega^{+} \delta_{\chi}\left(Q_{1}, \ldots, Q_{r}\right) \alpha_{\chi}^{+}\left(Q_{1}, \ldots, Q_{r}\right) z_{\chi},
$$

we see that

$$
\alpha_{\chi}^{+}\left(Q_{1}, \ldots, Q_{r}\right)=\frac{b^{2 r}}{\left|\operatorname{det} \Xi_{\chi}\right|^{2}} \alpha_{\chi}^{+}\left(P_{1}, \ldots, P_{r}\right) .
$$

Proposition 4.1. Assume the Birch-Swinnerton-Dyer and the Deligne-Gross conjectures. Then for any set $\left\{P_{1}, \ldots, P_{r}\right\}$ such that $\left\{P_{i}^{\sigma} \mid 1 \leq i \leq r, 1 \neq \sigma \in G\right\}$ generates a subgroup of finite index in $E_{\operatorname{Tr}}(K)$, the product

$$
\prod_{\chi_{0} \neq \chi \in \widehat{G}} \alpha_{\chi}^{+}\left(P_{1}, \ldots, P_{r}\right) \in \mathbb{Q}
$$

is a nonzero rational number.

Proof. This can be proved using arguments similar to those in the corresponding result [Fearnley and Kisilevsky 10, Proposition 7.2]. We briefly sketch the proof. By the Deligne-Gross conjecture, we have $r_{\chi}=r$ for all $\chi \neq \chi_{0}$, and so the Birch-SwinnertonDyer conjecture (1-1) implies

$$
0 \neq \prod_{\chi_{0} \neq \chi \in \widehat{G}} \frac{L^{(r)}(E, 1, \chi)}{r !}=\frac{R_{K}}{R_{\mathbb{Q}}} \times \frac{\left(\Omega^{+}\right)^{\ell-1}}{\sqrt{|d(K)|}} \times q_{1},
$$

where $0 \neq q_{1} \in \mathbb{Q}$. 
By $(2-3)$, we have

$$
\frac{R_{K}}{R_{\mathbb{Q}}}=\prod_{\chi_{0} \neq \chi \in \widehat{G}} \delta_{\chi}\left(P_{1}, \ldots, P_{r}\right) \times q_{2}
$$

with $0 \neq q_{2} \in \mathbb{Q}$. Also

$$
\begin{aligned}
& \prod_{\chi_{0} \neq \chi \in \widehat{G}} z_{\chi}=\prod_{\gamma \in \operatorname{Gal}(\mathbb{Q}(\chi) / \mathbb{Q})} z_{\chi^{\gamma}} \\
& =\prod_{\gamma \in \operatorname{Gal}(\mathbb{Q}(\chi) / \mathbb{Q})} \gamma\left(z_{\chi}\right) \in \mathbb{Q},
\end{aligned}
$$

and by [Washington 82, Corollary 4.6],

$$
\prod_{\chi \in \widehat{G}} \tau(\chi)=\prod_{\chi \in \widehat{G}} \frac{\mathfrak{f}_{\chi}}{\tau(\chi)}=\sqrt{|d(K)|},
$$

so it follows that

$$
0 \neq \prod_{\chi_{0} \neq \chi \in \widehat{G}} \alpha_{\chi}^{+}\left(P_{1}, \ldots, P_{r}\right) \in \mathbb{Q} .
$$

The similarity of formula $(4-1)$ to the formulas describing the Stark conjectures [Tate 84] and the results of the computations described in Section 5 suggest that the numbers $\alpha_{\chi}^{+}\left(P_{1}, \ldots, P_{r}\right)$ are algebraic and are in the real subfield $\mathbb{Q}(\chi)^{+}$of the cyclotomic field $\mathbb{Q}(\chi)$.

Conjecture 4.2. $\alpha_{\chi}^{+}\left(P_{1}, \ldots, P_{r}\right) \in \mathbb{Q}(\chi)^{+}$and

$$
\alpha_{\chi^{\gamma}}^{+}\left(P_{1}, \ldots, P_{r}\right)=\gamma\left(\alpha_{\chi}^{+}\left(P_{1}, \ldots, P_{r}\right)\right)
$$

for all $\gamma \in \operatorname{Gal}(\mathbb{Q}(\chi) / \mathbb{Q})$.

The result of Proposition 4.1 is a consequence of Conjecture 4.2 .

A more precise statement is predicted by the equivariant Tamagawa number conjecture in [Burns 10].

\section{NUMERICAL DATA}

It is our intention in this section to provide numerical data in support of Conjecture 4.2.

All curves are identified using the notation in [Cremona 92].

The values in Tables 2, 3, 5, 6 are expressed to six decimal places for ease of presentation. The original calculations were performed to between 15 and 30 decimal places. Most of the work was performed using PARI, with MAGMA used for the height calculations. ${ }^{1}$

\footnotetext{
${ }^{1}$ PARI is available at http://pari.math.u-bordeaux.fr/.
}

We first searched elliptic curves of conductor $N<40000$ for which there is a Dirichlet character $\chi$ of order 3 or 5 and of prime conductor $\mathfrak{f}_{\chi}<1000$ such that we have both $L(E, 1, \chi)=0$ and $L^{\prime}(E, 1, \chi)=0$. These candidate curves were then scanned for points over the corresponding number fields $K$ using a coarse search (looking for points in a small box). This usually failed, but nevertheless succeeded sufficiently often to provide illustrative data.

\subsection{Computational Methodology}

For a successful search, we have the following data: an elliptic curve $E / \mathbb{Q}$, a Dirichlet character $\chi$ of order 3 or 5 and conductor $\mathfrak{f}_{\chi}$ for which $L(E, 1, \chi)=0$, $L^{\prime}(E, 1, \chi)=0$, and points (of small height) in $E(K)$ for the cyclic extension $K / \mathbb{Q}$ cut out by the character $\chi$.

The next step is to determine from the set of discovered points, those pairs $P_{1}, P_{2}$ that are independent and can, together with their Galois conjugates, generate a subgroup of finite index in $E_{\operatorname{Tr}}(K)$. Each pair was tested by looking for a nonvanishing determinant of the heightpairing matrix described in Section 2.

The search produced examples satisfying all the above requirements. The value of the second derivative $L^{\prime \prime}(E, 1, \chi)$ was then computed for these cases using the Computel package described in [Dokchitser 04].

In the tables, it is important that the characters and the Galois action are fixed precisely.

Let $g$ be the smallest positive primitive root modulo $\mathfrak{f}_{\chi}$. (This is possible because we are considering only characters $\chi$ with prime conductor.) Then the Galois group $\operatorname{Gal}\left(\mathbb{Q}\left(\exp \left(2 \pi i / \mathfrak{f}_{\chi}\right) / \mathbb{Q}\right)\right.$ is generated by

$$
\sigma_{0}: \exp \left(2 \pi i / \mathfrak{f}_{\chi}\right) \mapsto \exp \left(2 \pi i g / \mathfrak{f}_{\chi}\right) .
$$

The field $K=K_{\mathfrak{f}_{\chi}}$ is the fixed field of $\sigma_{0}^{\ell}$ and is generated by

$$
t=\operatorname{Tr}_{\mathbb{Q}\left(\exp \left(2 \pi i / \mathfrak{f}_{\chi}\right)\right) / K}\left(\exp \left(2 \pi i / \mathfrak{f}_{\chi}\right)\right) .
$$

Fix the character $\chi_{\mathfrak{f}_{\chi}}$ by setting $\chi_{\mathfrak{f}_{\chi}}\left(\sigma_{0}\right)=\exp (2 \pi i / \ell)$.

By abuse of notation, we denote $\sigma_{0} \mid K$ by $\sigma_{0}$. For example, for the quintic subfield of conductor 11, we have $g=2, t=2 \cos (2 \pi / 11)$, and $\sigma_{0}(t)=t^{2}-2$, and $\chi\left(\sigma_{0}\right)=$ $\exp (2 \pi i / 5)$.

\begin{tabular}{lcc} 
Conductor & Minimal Polynomial & Galois Action \\
\hline 13 & $x^{3}+x^{2}-4 x+1$ & $t \rightarrow-t^{2}-2 t+2$
\end{tabular}

\section{TABLE 1.}




\begin{tabular}{c|cccc} 
Curve & Coefficients & $\mathfrak{f}_{\chi}$ & $L^{\prime \prime}\left(E, 1, \chi_{\mathfrak{f}_{\chi}}\right) / 2$ & $\tau(\chi)$ \\
\hline $427 \mathrm{~b} 1$ & {$[1,0,1,-8,7]$} & 13 & $4.824852+4.887124 i$ & $0.910836+3.488607 i$ \\
$420 \mathrm{c} 1$ & {$[0,1,0,-61,164]$} & 13 & $8.575300+2.356778 i$ & $0.910836+3.488607 i$
\end{tabular}

TABLE 2. Elliptic $L$-values cubic case.

\begin{tabular}{c|cccc} 
Curve & $\mathfrak{f}_{\chi}$ & Points & $\delta_{\chi}\left(P_{1}, P_{2}\right)$ & $\alpha_{\chi}^{+}\left(P_{1}, P_{2}\right)$ \\
\hline $427 \mathrm{~b} 1$ & 13 & {$\left[t, t^{2}-3\right] *,[t+1, t-1]$} & 2.683793 & 2.00000 \\
$420 \mathrm{c} 1$ & 13 & {$[t+3,3 t-4],\left[4 t^{2}+10 t+3,24 t^{2}+60 t-11\right]$} & 1.546242 & 12.000000
\end{tabular}

TABLE 3. Algebraic $L$-values cubic case.

\begin{tabular}{lll} 
Conductor & Minimal Polynomial & Galois Action \\
\hline 11 & $x^{5}+x^{4}-4 x^{3}-3 x^{2}+3 x+1$ & $t \rightarrow t^{2}-2$ \\
31 & $x^{5}+x^{4}-12 x^{3}-21 x^{2}+x+5$ & $t \rightarrow\left(3 t^{4}-t^{3}-33 t^{2}-24 t+15\right) / 5$
\end{tabular}

TABLE 4. Cyclic quintic fields.

\begin{tabular}{c|cccl} 
Curve & Coefficients & $\mathfrak{f}_{\chi}$ & $L^{\prime \prime}\left(E, 1, \chi_{\mathfrak{f}_{\chi}}\right) / 2$ & $\tau(\chi)$ \\
& & & $L^{\prime \prime}\left(E, 1, \chi_{\mathfrak{f}_{\chi}}^{\gamma}\right) / 2$ & $\tau\left(\chi^{\gamma}\right)$ \\
\hline $1299 \mathrm{c} 1$ & {$[1,0,0,-10,11]$} & 31 & $-0.196900+13.286137 i$ & $4.55242-3.20554 i$ \\
& & & $16.670545+0.629368 i$ & $5.22658+1.91908 i$ \\
$6355 \mathrm{e} 1$ & {$[0,0,1,-17,27]$} & 11 & $12.724729+18.419497 i$ & $2.63611+2.01270 i$ \\
& & & $10.288195-0.471113 i$ & $2.07016+2.59122 i$ \\
$13488 \mathrm{c} 1$ & {$[0,1,0,-23,36]$} & 11 & $18.922505-5.654659 i$ & $2.63611+2.01270 i$ \\
& & & $16.494633+10.867191 i$ & $2.07016+2.59122 i$ \\
$17747 \mathrm{c} 1$ & {$[1,-1,1,-15,26]$} & 11 & $7.594316-21.610790 i$ & $2.63611+2.01270 i$ \\
& & & $29.627304+8.148574 i$ & $2.07016+2.59122 i$ \\
$25551 \mathrm{~b} 1$ & {$[0,0,1,-24,43]$} & 11 & $29.182911-8.720802 i$ & $2.63611+2.01270 i$ \\
& & & $12.886158+8.489812 i$ & $2.07016+2.59122 i$
\end{tabular}

TABLE 5. Elliptic $L$-values quintic case.

\begin{tabular}{|c|c|c|c|c|}
\hline Curve & $\mathfrak{f}_{\chi}$ & Points & $\begin{array}{l}\delta_{\chi}\left(P_{1}, P_{2}\right) \\
\delta_{\chi^{\gamma}}\left(P_{1}, P_{2}\right)\end{array}$ & $\begin{array}{l}\alpha_{\chi}^{+}\left(P_{1}, P_{2}\right) \\
\alpha_{\chi^{\gamma}}^{+}\left(P_{1}, P_{2}\right) \\
\end{array}$ \\
\hline $1299 \mathrm{c} 1$ & 31 & $\begin{array}{l}{\left[-t,\left(2 t^{4}+t^{3}-22 t^{2}-31 t\right) / 5\right] *} \\
{\left[t+1,-t^{4}+t^{3}+10 t^{2}+3 t-4\right]}\end{array}$ & $\begin{array}{l}1.776198 \\
3.608210\end{array}$ & $\begin{array}{l}-16.180340 \\
6.180340\end{array}$ \\
\hline $6355 \mathrm{e} 1$ & 11 & $\begin{array}{c}{\left[t+2, t^{4}-t^{3}-2 t^{2}+4 t-1\right]} \\
{\left[-t^{2}-t+2,3 t^{3}+2 t^{2}-6 t-3\right] *}\end{array}$ & $\begin{array}{l}1.837063 \\
1.367420\end{array}$ & $\begin{array}{l}5.000000 \\
5.000000\end{array}$ \\
\hline $13488 \mathrm{c} 1$ & 11 & $\begin{array}{c}{\left[t^{3}-2 t+1,2 t^{4}+2 t^{3}-5 t^{2}-7 t+1\right]} \\
{\left[t^{3}+2 t^{2}+2,4 t^{4}+8 t^{3}-4 t^{2}-9 t-1\right] *}\end{array}$ & $\begin{array}{l}1.065590 \\
4.514680\end{array}$ & $\begin{array}{c}13.090170 \\
1.909830\end{array}$ \\
\hline $17747 \mathrm{c} 1$ & 11 & $\begin{array}{l}{\left[-t^{2}-2 t+2,-t^{4}-2 t^{3}+3 t^{2}+7 t-1\right] *} \\
{\left[-t^{3}+t+2,-2 t^{4}+3 t^{3}+7 t^{2}-4 t-5\right] *}\end{array}$ & $\begin{array}{c}1.510702 \\
13.889945\end{array}$ & $\begin{array}{c}-21.180340 \\
1.180340\end{array}$ \\
\hline 25551b1 & & $\begin{array}{c}{\left[3 t+1,-3 t^{4}+15 t^{2}-8\right]} \\
{\left[t^{3}-2 t+3,2 t^{4}+t^{3}-5 t^{2}-2 t+1\right] *}\end{array}$ & $\begin{array}{c}2.671241 \\
15.009137\end{array}$ & $\begin{array}{c}18.944272 \\
1.055728\end{array}$ \\
\hline
\end{tabular}

TABLE 6. Algebraic $L$-values quintic case. 
Similarly, to fix the action of $\operatorname{Gal}(\mathbb{Q}(\chi) / \mathbb{Q})$, let $b$ denote the smallest positive primitive root modulo $\ell$ and define $\gamma \in \operatorname{Gal}(\mathbb{Q}(\chi) / \mathbb{Q})$ by $\gamma(\zeta)=\zeta^{b}$ for all $\ell$ th roots of unity $\zeta$. Then $\chi^{\gamma}(\sigma)=\gamma(\chi(\sigma))$ for all $\chi \in \widehat{G}$ and all $\sigma \in G$.

\subsection{Cyclic Cubic Extensions}

The number field corresponds to the Dirichlet character of conductor 13 with the relevant properties shown in Table 1.

Table 2 shows the basic properties of the elliptic curves, the values of the derivatives of the critical $L$ functions, and the value of the Gauss sums.

The data on the points are shown in Table 3, where $t$ is a root of the minimal polynomial indicated above. It should be noted that the points are not necessarily the generators of the Mordell-Weil group.

The values of $\alpha_{\chi}^{+}(P)$ for the above two curves correspond to at least 15 decimal places to integers and therefore appear to lie in the real subfield of the field of cube roots of unity.

It should be noted that the point shown in Table 3 with an asterisk does not have zero trace. In this case, the point was differenced with its Galois conjugate and the modified point was then used in the subsequent analysis to compute the alpha value.

\subsection{Cyclic Quintic Extensions}

The number fields correspond to Dirichlet characters of conductors 11 and 31 with relevant properties as given in Table 4.

Table 5 shows the basic properties of the elliptic curves, the values of the derivatives of the critical $L$ functions, and the value of the Gauss sums.

The data on the points are shown in Table 6, where $t$ is a root of the minimal polynomial indicated above. It should be noted that the points are necessarily the generators of the Mordell-Weil group.

The values of $\alpha_{\chi}^{+}\left(P_{1}, P_{2}\right)$ for the five curves shown in Table 6 correspond to at least 15 decimal places to the roots of

$$
\begin{aligned}
& x^{2}+10 x-100=0 \text { for } 1299 \mathrm{c} 1 \\
& x^{2}-10 x+25=0 \text { for } 6355 \mathrm{e} 1 \\
& x^{2}-15 x+25=0 \text { for } 13488 \mathrm{c} 1
\end{aligned}
$$

$$
\begin{aligned}
& x^{2}+20 x-25=0 \text { for } 17747 \mathrm{c} 1 \\
& x^{2}-20 x+20=0 \text { for } 25551 \mathrm{~b} 1
\end{aligned}
$$

All these roots lie in the maximal real subfield of the field of fifth roots of unity.

It should be noted that the points shown in the table with an asterisk do not have zero trace. In these cases, the points were differenced with their Galois conjugates, and these modified points were then used in the subsequent analysis to compute the alpha values.

\section{ACKNOWLEDGMENTS}

This work was supported in part by a grant from NSERC.

\section{REFERENCES}

[Bley 10] W. Bley. "Numerical Evidence for the Equivariant Birch and Swinnerton-Dyer Conjecture." Exp. Math. 20:4 (2011), 426-456.

[Burns 10] David Burns. "Leading Terms and Values of Equivariant Motivic L-Functions." Pure App. Math. Q. 6 (2010), pp. 83-171 (John Tate special issue, Part II).

[Burns and Flach 01] D. Burns and M. Flach. "Tamagawa Numbers for Motives with (Noncommutative) Coefficients." Documenta Math. 6 (2001), 501570 .

[Cremona 92] J. E. Cremona. Algorithms for Modular Elliptic Curves. Cambridge University Press, 1992.

[Deligne 79] P. Deligne. "Valeurs de fonctions $L$ et périods d'intégrales." In Automorphic Forms, Representations, and L-Functions, Proc. Symp. Pure Math. 33, part 2, pp. 313-346. Amer. Math. Soc., 1979.

[Dokchitser 04] T. Dokchitser. "Computing Special Values of Motivic L-Functions." Exp. Math. 13:2 (2004), 137-150.

[Dokchitser and Dokchitser 10] T. Dokchitser and V. Dokchitser. "On the Birch-Swinnerton-Dyer Quotients Modulo Squares." Annals of Math. 172:1 (2010), $567-596$.

[Fearnley and Kisilevsky 10] J. Fearnley and H. Kisilevsky. "Critical Values of Derivatives of Twisted Elliptic $L$ Functions." Exp. Math. 19:2 (2010), 149-160. 
[Tate 84] J. Tate. Les Conjectures de Stark sur les Fonctions $L$ d'Artin en $s=0$, Progress in Mathematics 47. Birkhäuser, 1984.
[Washington 82] L. Washington, L. Introduction to Cyclotomic Fields, Graduate Texts in Mathematics 83. Springer, 1982.

Jack Fearnley, Department of Mathematics and Statistics and CICMA, Concordia University, 1455 de Maisonneuve Blvd. West, Montréal, Quebec, H3G 1M8, Canada (jack@mathstat.concordia.ca)

Hershy Kisilevsky, Department of Mathematics and Statistics and CICMA, Concordia University, 1455 de Maisonneuve Blvd. West, Montréal, Quebec, H3G 1M8, Canada, (kisilev@mathstat.concordia.ca) 